\title{
Evaluación de un protocolo para el control de la vasectomía y modificaciones a realizar
}

\author{
Cháfer Rudilla $\mathrm{M}^{*}$, Navarro Casado L*, Quintanilla Mata ML*, Belilty Araque $\mathrm{M}^{*}$, \\ Andrés Fernández C*, Salinas Sánchez J**.
}

*Laboratorio de Análisis Clínicos. ** Servicio de Urología. Complejo Hospitalario y Universitario de Albacete.

Actas Urol Esp. 2007;31(5):488-492

\section{RESUMEN}

EVALUACIÓN DE UN PROTOCOLO PARA EL CONTROL DE LA VASECTOMÍA Y MODIFICACIONES A REALIZAR

Objetivo: Revisar los resultados de la implantación de un protocolo para el control de la vasectomía en el laboratorio del Hospital General de Albacete y realizar algunas modificaciones que nos permitan reducir el número de controles que deben realizarse nuestros pacientes, pero que esté en concordancia con las recomendaciones internacionales.

Material y Métodos: Se revisaron los controles de las vasectomías realizadas desde Enero de 2002 a Diciembre de 2004. Nuestro protocolo consiste en una adaptación a nuestro medio de las guías de la Sociedad Británica de Andrología (BAS) para el control de la vasectomía y de la Organización Mundial de la Salud para el análisis seminal.

Resultados: Durante los 3 años del estudio se han intervenido 984 pacientes de vasectomía, de los cuales el 67\% ha acudido a nuestro laboratorio a realizarse algún control, pero solamente un 55,5\% de los mismos cumplió los criterios de alta establecidos; el resto, abandonó antes de conseguirlo. Se recibió una media de 2 muestras por paciente, aunque un $39.6 \%$ de los mismos sólo trajo una y más del 23\% trajeron más de 3 . Tuvimos 43 fallos de la cirugía (5,9\%), 4 casos de recanalización precoz $(0,5 \%)$ y uno de recanalización tardía $(0,1 \%)$, y un total de 13 pacientes fueron reintervenidos $(1,7 \%)$.

Conclusiones: Un porcentaje elevado de pacientes vasectomizados no cumplió los criterios de alta recomendados por la BAS y casi la cuarta parte de los mismos debe realizar más de 3 controles. Por este motivo, creemos conveniente modificar nuestro protocolo para control de la vasectomía y proponer que los pacientes reciban en la consulta dos volantes de solicitud de espermiograma para los meses $6^{\circ}$ y $7^{\circ}$ tras la cirugía y que acudan de nuevo para la valoración conjunta de ambos resultados. En este momento, con tan solo 2 controles, la mayoría de los pacientes estarán en condiciones de conseguir el alta. Aquellos en los que se produjo un fallo de la cirugía o sufrieron una recanalización precoz, con una segunda muestra de confirmación, podrán ser derivados al urólogo sin mayor dilación.

Palabras Clave: Control de vasectomía. Protocolo de vasectomía. Recanalización.

\section{ABSTRACT \\ EVALUATION OF A GUIDELINES FOR THE ASSESSMENT OF SUCCESS AFTER VASECTOMY AND NEEDED IMPRO- VEMENTS}

Objectives: The evaluation of a recently established guidelines about the assessment of semen samples after vasectomy in the laboratory of the Hospital General of Albacete and to modify them to optimize the number of semen samples provided per patient but keeping in concordande with the international recommendations.

Patients and Methods: The records of seminal analysis results from vasectomies performed from January 2002 to December 2004 were reviewed. Our vasectomy guidelines are based upon those of the British Andrology Society (BAS) and those of the World Health Organization for seminal assessment.

Results: During the 3 years 984 patients underwent vasectomy. At follow up, $67 \%$ of them returned postvasectomy semen samples, but just 55,5\% of them get the clearance criteria; the other patient abandoned before getting them. A mean of 2 samples per patient were received, but 39,6\% of them provided one and more than $23 \%$ brought more than 3 semen samples. We had 43 technical failures, 4 early recanalization $(0,5 \%)$ and one late recanalization $(0,1 \%)$, and 13 patients underwent revasectomy $(1,7 \%)$.

Conclusion: A high percentage of our patients failed to fulfill the clearance criteria recommended by the BAS and almost a quarter of them had to deliver more than 3 semen samples. So we find convenient to modify our guides and propose that our patients should receive 2 request forms from the practitioner for semen analysis in the 6 th and the 7 th month postoperatively and should return to global evaluation of both reports. In that moment most patients will be able to meet the applied criteria for success with just 2 semen samples. Those who fail to become sterile because of either technical failure or early recanalizatione may be advised to go to the urologist with just 2 semen analysis without unnecesary delay.

Keywords: Vasectomy assessment. Vasectomy guidelines. Recanalization. 
$\mathrm{R}$ ecientemente, hemos implantado en nuestro aboratorio del Hospital General de Albacete un protocolo para el control de la vasectomía ${ }^{1}$, basado en las recomendaciones de la Sociedad Británica de Andrología (BAS) ${ }^{2}$ y las de recogida de semen para estudio de fertilidad de la Organización Mundial de la Salud $(\mathrm{OMS})^{3}$. En dicho protocolo se aconseja iniciar los controles a las 16 semanas de la intervención, después de haber realizado, al menos, 24 eyaculaciones. Se debe examinar al microscopio una alícuota del semen fresco y, en caso de no observar espermatozoides, volver a analizar el semen tras centrifugación. Se recomienda dar el alta al paciente después de conseguir dos resultados negativos consecutivos. Cuando de forma persistente se visualice un pequeño número de espermatozoides muertos, el paciente puede ser dado de alta una vez transcurridos 7 meses desde la cirugia; esto se basa en que en ese momento, según los estudios, la probabilidad de gestación es similar a la de los pacientes con un resultado negativo ${ }^{4}$. Según estas guías, si un paciente presenta espermatozoides residuales puede llegar a tener que realizarse 4 controles antes de conseguir el alta, suponiendo que trajera una muestra por mes. Esto representa un considerable número de espermiogramas a realizar por el laboratorio, con las consiguientes consultas médicas de seguimiento, sin olvidar la desconfianza que crea en los pacientes.

El objetivo de nuestro trabajo fue revisar nuestros resultados y valorar alguna modificación del protocolo que nos permitiera reducir el número de controles que deben realizarse nuestros pacientes, pero que estuviera en concordancia con las recomendaciones internacionales.

\section{MATERIAL Y MÉTODOS}

Descargamos del Sistema de Información del Laboratorio Omega 2000 los datos de todos los controles de vasectomía correspondientes a las intervenciones realizadas entre enero de 2002 y diciembre de 2004; para conocer el número de vasectomías incluidas en dicho periodo se consultó con la documentalista del Hospital.

El protocolo de análisis seminal se basa en las guías para el control de la vasectomía de la BAS que, a su vez, se fundamenta en las de las ins- trucciones para la recogida seminal para el estudio de fertilidad de la OMS.

Las variables categóricas se muestran como número y porcentaje; las variables continuas se expresan como media \pm desviación estándar. Se empleó la prueba de Kolmogorov-Smirnov para comprobar la normalidad de las variables continuas.

\section{RESULTADOS}

Durante el periodo del estudio, 984 pacientes fueron sometidos a la intervención de vasectomía, de los cuales, $664(66,4 \%)$ trajeron muestras de semen para control. Además, incluimos en el estudio a 67 pacientes que se realizaron los espermiogramas en nuestro laboratorio y que procedian de cirugias practicadas en otros centros.

En total, se analizaron 1430 muestras de semen de los 731 pacientes que se realizaron controles, con una media de 2.0 muestras por paciente analizado. El número de seminogramas efectuados por paciente se refleja en la Tabla 1 .

Tabla 1

Número de controles realizados por paciente

\begin{tabular}{ccc}
\hline Número controles & $\mathbf{N}$ & $\%$ \\
\hline 1 & 731 & 100,0 \\
2 & 441 & 60,3 \\
3 & 165 & 22,5 \\
4 & 57 & 7,8 \\
5 & 20 & 2,7 \\
6 & 8 & 1,1 \\
7 & 2 & 0,3 \\
9 & 1 & 0,1 \\
\hline
\end{tabular}

Los resultados del primer control, realizado a las $16 \pm 9,3$ semanas, se muestran en la Tabla 2 . Hubo 449 pacientes $(61,4 \%)$ en los que no se observaron espermatozoides en este primer control, pero 231 (31,6\% del total) no trajeron la segunda muestra para confirmar la azoospermia. De los pacientes que sí trajeron un nuevo eyaculado, 196 (43,7\%) obtuvieron un resultado también negativo; $14(3,1 \%)$ mostraron espermatozoides en su sedimento y $4(0,9 \%)$, en el semen sin centrifugar; 3 varones sufrieron recanalización. 
Tabla 2

Resultados del primer control de la vasectomía

\begin{tabular}{lcccc}
\hline Recuento (espermatozoides/mL) & $\mathrm{N}$ & $\%$ & Móviles (N) & Vitalidad positiva (N) \\
\hline Azoospermia & 414 & 56,6 & - & - \\
No se observan espermatozoides* & 35 & 4,8 & - & - \\
$100-1000$ & 122 & 16,7 & - & - \\
$1000-5000$ & 111 & 15,2 & - & 1 \\
$10000-10^{5}$ & 16 & 2,2 & 3 & 3 \\
$10^{5}-10^{6}$ & 8 & 1,1 & 2 & 4 \\
$>10^{6}$ & 20 & 2,7 & 11 & - \\
Muestras rechazadas** & 5 & 0,7 & - & 11 \\
Total & 731 & 100 & 16 &
\end{tabular}

* No se podía informar azoospermia debido a la presencia de abundante respuesta inflamatoria o esmegma.

** Muestras recibidas en contenedores inadecuados o derramadas.

Cincuenta y tres de los 731 pacientes (5,9\%) tuvieron frecuentes espermatozoides móviles en todos sus espermiogramas y fueron considerados fallos de la cirugía. Nos encontramos 4 casos de recanalización precoz $(0,5 \%)$, considerando como tal la que se produce en el período inferior a lo 6 meses tras la cirugía y sin haber conseguido el alta. Los resultados de los dos primeros controles de cada uno de estos pacientes se indican en la Tabla 3. Además, tuvimos un caso de recanalización tardía, que dio lugar a una gestación inesperada, en un paciente que había conseguido el alta con un resultado de azoospermia; en su eyaculado se observaron 7 millones $/ \mathrm{mL}$ de espermatozoides, frecuentes de ellos móviles, con un $34 \%$ de vitalidad positiva.

Se realizaron 13 reintervenciones de vasectomía $(1,7 \%)$. En la Tabla 4 se muestran los recuentos que motivaron la nueva cirugía, así como los resultados de los controles posteriores a la intervención.

De los 731 pacientes estudiados, $406(55,5 \%)$ cumplieron los criterios de alta recomendados por la BAS, según queda reflejado en la Tabla 5 . En la Tabla 6 se muestran los motivos por los que 325 pacientes $(44,5 \%)$ no cumplieron dichos criterios.

Tabla 3

Recanalizaciones precoces

Tabla 4

\section{DISCUSIÓN}

Como se desprende de nuestros resultados, 731 pacientes (66\%) de los 1052 que fueron sometidos a la intervención de vasectomía durante los 3 años del estudio han traído muestras de semen para su control, porcentaje similar al de otros estudios con un diseño semejante ${ }^{5,6}$.

Aunque en nuestro protocolo se recomienda dar el alta a los pacientes tras dos controles consecutivos negativos, sólo 442 varones $(60,4 \%)$ trajeron un segundo eyaculado; es más, de los $449(61,4 \%)$ que obtuvieron azoospermia en su primer control, ésta no fue confirmada en 226 (50,3\%); a éstos hay que añadir otros 9 pacientes que, a lo largo de sus controles, obtuvieron un resultado negativo y no lo confirmaron.

A pesar de la reticencia de nuestros pacientes a realizarse más de un control, lo consideramos un requerimiento incuestionable de nuestro protocolo. Como demostramos en un trabajo anterior, sólo la frecuente incidencia de problemas preanalíticos que encontramos, como la recogida de un eyaculado incompleto, o analíticos, como

\begin{tabular}{ccccc}
\hline Paciente & $\begin{array}{c}\text { Primer control } \\
\text { Semana }\end{array}$ & $\begin{array}{c}\text { Secuento } \\
\text { (espermatozoides/mL) }\end{array}$ & Semana & $\begin{array}{c}\text { Secuento } \\
\text { (espermatozoides /mL) }\end{array}$ \\
\hline 1 & 10 & $1000-5000$ & 14 & $1 \times 10^{6}$ \\
2 & 9 & Azoospermia & 25 & $32 \times 10^{6}$ \\
3 & 11 & Azoospermia & 20 & $70 \times 10^{6}$ \\
4 & 14 & Azoospermia & 23 & $1 \times 10^{5}$ \\
\hline
\end{tabular}

Reintervenciones realizadas y recuentos posteriores a la intervención

\begin{tabular}{lclc}
\hline Motivo de la reintervención & N & Recuento al alta post reintervención & N \\
Abundantes espermatozoide móviles & 8 & Azoospermia & 4 \\
& & Azoospermia/<10000esp inmóviles $/ \mathrm{mL}$ & 4 \\
$<5 \times 10^{5} \mathrm{esp}$ inmóviles/mL & 2 & $1000-5000 \mathrm{esp}$ inmóviles $/ \mathrm{mL}$ & 2 \\
$1000-5000 \mathrm{esp}$ inmóviles $/ \mathrm{mL}$ & 3 & $1000-5000 \mathrm{esp}$ inmóviles $/ \mathrm{mL}$ & 3 \\
Total & 13 & & 13 \\
\hline
\end{tabular}


Tabla 5

Pacientes que cumplieron los criterios de alta

\begin{tabular}{llrrc}
\hline Criterio de alta alcanzado & Recuento (espermatozoides/mL) & N & \% & \% total de pacientes \\
\hline Alta ideal & Azoospermia & 242 & 59,6 & 33,1 \\
Alta especial & Azoospermia/<10000 & 119 & 29,3 & 16,3 \\
& $<10000$ varias ocasiones $>7$ meses & 45 & 11,1 & 6,2 \\
Total & & 406 & 100,0 & 55,5 \\
\hline
\end{tabular}

Tabla 6

Pacientes que no cumplieron los criterios de alta y motivos por los que no lo hicieron

\begin{tabular}{llccc}
\hline $\begin{array}{l}\text { Cumplimiento } \\
\text { criterios de alta }\end{array}$ & $\begin{array}{l}\text { Recuento } \\
\text { (espermatozoides/mL) }\end{array}$ & N & \% N* & \% del total** \\
\hline Cumplimiento parcial & Azoospermia no confirmada & 236 & 72,6 & 32,3 \\
& $<10000$ no confirmado o <6 meses & 75 & 23,1 & 10,3 \\
No cumplimiento & Abundantes espermatozoides móviles & 6 & 1,8 & 0,8 \\
& Abundantes espermatozoides inmóviles & 7 & 2,2 & 1,0 \\
Total & Semen en contenedor inadecuado & 1 & 0,3 & 0,1 \\
\hline
\end{tabular}

* Porcentaje respecto de los 325 pacientes que no cumplieron los criterios de alta.

** Porcentaje respecto de los 731 pacientes que se realizaron los controles de vasectomía.

alteraciones en la consistencia o una respuesta inflamatoria más o menos intensa, justifican la necesidad de obtener un mínimo de dos espermiogramas por paciente ${ }^{7}$.

Sin embargo, tampoco podemos olvidar que, fundamentalmente debido a la presencia de espermatozoides residuales, casi la cuarta parte de los pacientes $(22,5 \%)$ tuvieron que traer 3 muestras de semen o más. Aunque existe controversia en cuanto al momento ideal para iniciar los controles ${ }^{8,9}$, numerosos autores se inclinan por retrasarlo hasta los 6 meses de la intervención para intentar reducir el número de especimenes. Smith et $\mathrm{al}^{9}$ compararon los resultados de comenzar el análisis seminal a los 3 o a los 6 meses de la cirugía y optaron por el segundo intervalo, ya que supone la realización de un número menor de espermiogramas por paciente y la mayoría de los varones no mostró preferencias por uno u otro período. Gómez de Vicente et $\mathrm{al}^{10}$, después de analizar sus resultados, empezaron a solicitar el primer seminograma a los 7 meses de la vasectomía, siendo este nuevo régimen bien aceptado por los pacientes. Nosotros, a la vista de nuestros resultados, nos planteamos si retrasar el inicio de los controles a los 6 meses de la intervención podría reducir el número de seminogra- mas a realizar. Evidentemente, en ese momento, no hubiéramos podido diferenciar el grupo de pacientes recanalizados precozmente de aquellos en los que se han producido verdaderos fallos de la cirugía. Aunque es difícil conocer el momento exacto en el que se produjeron las recanazaciones, como se observa en la Tabla 3, en nuestros 4 pacientes fue antes de los 6 meses post intervención. En cuanto a los fallos de la cirugía, que afectaron a 43 pacientes (5,9\%), hubieran sido identificados en cualquier momento, ya que en todos los controles realizados se observaron abundantes espermatozoides móviles. Según Escho et $\mathrm{al}^{11}$, a los 6 meses de la intervención es raro un cambio favorable en la evolución de un paciente que presenta espermatozoides móviles, por eso éste es el momento ideal para detectar ambos problemas. Por otro lado, el no poder filiar la causa del fracaso no va a reportar ningún beneficio para el paciente y sí supone un mayor gasto sanitario difícilmente justificable.

En definitiva, a los 6 meses de la intervención, según nuestros resultados, la mayoría de los pacientes estarán en condiciones de conseguir el alta con sólo 2 espermiogramas y aquellos en los que la intervención no haya tenido éxito, tampoco deberán traer más de dos muestras. 
En contrapartida, para intentar conseguir que un mayor número de pacientes consiga el alta, hemos decidido proponer que el médico de cabecera les entregue, en el momento en que acudan a su consulta, dos volantes de solicitud de espermiograma para control de la vasectomía para el $6^{\circ}$ y el $7^{\circ}$ mes tras la intervención. De esta manera, seguiríamos cumpliendo con los criterios de alta más extendidos actualmente, ahorrariamos visitas médicas de seguimiento $\mathrm{y}$, en el momento en que acuda a la revisión de los resultados, en un porcentaje muy elevado de los casos, se le podría dar el alta al paciente.

\section{CONCLUSIONES}

Nuestros resultados muestran que casi la cuarta parte de los pacientes que acuden a realizarse los controles de vasectomía debe traer más de 3 muestras y que sólo el 55,5\% del total cumple los criterios de alta aconsejados por la BAS. Por este motivo, hemos decidido modificar nuestras recomendaciones y proponer que los pacientes reciban en la consulta dos volantes de solicitud de espermiograma para los meses $6^{\circ}$ y $7^{\circ}$ post intervención y que acudan a la consulta para la valoración conjunta de ambos resultados. De esta manera, la mayoría de los pacientes conseguirá el alta con sólo los 2 espermiogramas imprescindibles para cumplir las recomendaciones internacionales y todo redundará en una disminución del gasto sanitario. Por supuesto, se necesitan estudios posteriores para confirmar que estas medidas cumplen los objetivos propuestos.

\section{REFERENCIAS}

1. Cháfer M, Navarro L, Fuster O, Andrés C, Vera J, Domínguez JP. Protocolo para el control de la vasectomía. Revista de Diagnóstico Biologico. 2006;55(1):55-59.

2. Hancock P, McLaughlin E. British Andrology Society guidelines for the assessment of postvasectomy semen samples (2002). J Clin Pathol. 2002;55(11):812-816.

3. WHO, Laboratory manual for the examination of human semen and sperm cervical mucus interaction, 4th ed. Cambridge; Cambridge University Press, UK, 1999.

4. Benger JR, Swami SK, Gingell C. Persistent spermatozoa after vasectomy: a survey of British urologist. Br J Urol. 1995;76(3):376-379.

5. Smucker DR, Mayhew HE, Nordlund DJ, Hahn WK Jr, Palmer KE. Postvasectomy semen analysis: why patients don't follow-up. J AM Board Fam Pract. 1991;4:5-9.

6. Maatman TJ, Aldrin L, Carothers GG. Patient noncompliance after vasectomy. Fertil Steril. 1997;68(3):552-555.

7. Cháfer M, Navarro L, Belilty M, Andrés C, Quintanilla M. Influencia del proceso analítico en la aparición y desaparición de los espermatozoides del semen tras la vasectomía.

8. Edwards IS. Earlier testing after vasectomy, based on the absence of motile sperm. Fertil Steril. 1993;59(2):431-436.

9. Smith AG, Crooks J, Singh NP et al. Is the timing of postvasectomy seminal analysis important? Br J Urol. 1998;81 (3):458-460.

10. Gómez de Vicente JM, Romero I, Blanco C, Pastor J, Moreno A, Santos D, Miravalles E, Berenguer A. La historia natural del aclaramiento de espermatozoides en el semen tras la vasectomía. Actas Urol Esp. 2004;28(4):286289.

11. Escho JO, Cass AS. Recanalization rate following methods of vasectomy using interposition of fascial sheath of vas deferens. J Urol. 1978;120(2):178-179.

Correspondencia autor: Dra. M. Cháfer Rudilla Servicio de Análisis Clínicos

Complejo Hospitalario y Universitario de Albacete

Hermanos Falcó, 37. 02006

E-mail autora: mcharud@telefonica.net

Información del trabajo: Original - Andrologia

Trabajo recibido: marzo 2007

Trabajo aceptado: abril 2007 